\title{
Unusual Emissions at Various Energies Prior to the Impulsive Phase of the Large Solar Flare and Coronal Mass Ejection of 4 November 2003
}

\author{
Pierre Kaufmann • Gordon D. Holman • Yang Su • C. Guillermo Gimenez de Castro • \\ Emilia Correia • Luis O.T. Fernandes • Rodney V. de Souza • Adolfo Marun • \\ Pablo Pereyra
}

Received: 23 March 2011 / Accepted: 17 May 2012 / Published online: 3 July 2012

(C) Springer Science+Business Media B.V. 2012

\begin{abstract}
The GOES X28 flare of 4 November 2003 was the largest ever recorded in its class. It produced the first evidence for two spectrally separated emission components, one at microwaves and the other in the THz range of frequencies. We analyzed the pre-flare phase of this large flare, twenty minutes before the onset of the major impulsive burst. This period is characterized by unusual activity in X-rays, sub-THz frequencies, $\mathrm{H} \alpha$, and microwaves. The CME onset occurred before the onset of the large burst by about $6 \mathrm{~min}$. It was preceded by pulsations of $3-5 \mathrm{~s}$ periods at sub-THz frequencies together with $\mathrm{X}$-ray and microwave enhancements. The sub-THz pulsations faded out as impulsive bursts were detected at $100-$ $300 \mathrm{keV}$ and $7 \mathrm{GHz}$, close to the time of the first $\mathrm{H} \alpha$ brightening and the CME onset. The activities detected prior to and at the CME onset were located nearly 2 arcmin south of the
\end{abstract}

P. Kaufmann $(\bowtie) \cdot$ C.G. Gimenez de Castro · E. Correia · L.O.T. Fernandes · R.V. de Souza Escola de Engenharia, CRAAM, Universidade Presbiteriana Mackenzie, São Paulo, SP 01302970, Brazil

e-mail: kaufmann@craam.mackenzie.br

P. Kaufmann

Centro de Componentes Semicondutores, CCS, Universidade Estadual de Campinas, Campinas, SP 13083970, Brazil

G.D. Holman · Y. Su

National Aeronautics and Space Administration, Goddard Space Flight Center, Code 671, Greenbelt, MD 20771, USA

Y. Su

Department of Physics, The Catholic University of America, Washington, DC 20064, USA

Y. Su

Purple Mountain Observatory, Chinese Academy of Sciences, Nanjing 210008, China

E. Correia

Instituto Nacional de Pesquisas Espaciais', São José dos Campos, SP 12201970, Brazil

A. Marun · P. Pereyra

Complejo Astronômico El Leoncito, CONICET, San Juan, Argentina 
following large flare, suggesting they were separate events. This unusual activity brings new clues to understanding the complex energy buildup mechanisms prior to the CME onset, occurring at a distinct location and well before the major flare that exploded afterwards.

Keywords Coronal mass ejections (CMEs) $\cdot$ CME precursors $\cdot$ Solar flares $\cdot$ Sub-THz emissions $\cdot \mathrm{X}$-ray emissions

\section{Introduction}

The relationships between coronal mass ejections (CMEs) and flares remain unclear, mainly because there are a variety of situations that prevent a single explanation (see the review by Mittal and Narain (2010) and references therein). The initiation phase of CME events is not well known in most cases, because most coronagraphs cannot observe the source region in the solar atmosphere at lower altitudes. However, the analysis of a few events at lower altitudes in the solar atmosphere has suggested a two-phase CME launch process (Zhang et al., 2001). The initiation phase starts at slow speeds (tens of $\mathrm{km} \mathrm{s}^{-1}$ ), tens of minutes before the acceleration (or onset) phase, exhibiting higher speeds (hundreds to a few thousand $\mathrm{km} \mathrm{s}^{-1}$ ). The onset phase begins at altitudes lower than those in available coronagraph observations.

There are indications that the CME onset phase is associated with weak soft X-ray enhancements, sometimes preceding larger X-ray bursts (Datlowe, Elcan, and Hudson, 1974; Harrison et al., 1985; Gopalswamy et al., 2006; Schwenn et al., 2006). For a number of events analyzed it has been found that there were sub-THz pulsations associated with the CME onset phase (Kaufmann et al., 2003). On the other hand, there are CMEs occurring without any obvious flare connection (Webb, 2000). There have been studies suggesting that solar flares are a consequence of CMEs rather than causing them. Namely, the expansion of a CME loop may subsequently trigger magnetic reconnection, giving rise to a flare afterwards. This view is consistent with a general tendency that the CME onset precedes the flare (Harrison et al., 1985; Harrison, 1986; Kahler, 1992). On the other hand it has been suggested that both the CME initiation and the pre-flare phases involve ejection and heating of material by magnetic reconnection, and therefore they may all be part of a common dynamic instability process (Ohyama and Shibata 1997, 1998).

On 4 November 2003, the X-ray intensity of the flare started to increase at about 19:32 UT, and peaked between 19:40 - 19:50 UT. The major microwave and sub-THz intensities rose at about 19:40 UT (Kaufmann et al., 2004). These start times were derived as the time when intensities rose and became detectable, and therefore, they contain an uncertainty of several minutes. This flare is known as the largest soft X-ray burst ever recorded (extrapolated GOES class X28 to X48, after Woods et al. (2004), Thomson, Rodger, and Dowden (2004), and Brodrick, Tingay, and Wieringa (2005)) and for producing the first evidence for a $\mathrm{THz}$ emission component spectrally distinct from the well-known microwave emission (Kaufmann et al., 2004). The Large Angle and Spectrometric Coronagraph (LASCO) instrument (Brueckner et al., 1995) onboard the Solar and Heliospheric Observatory (SOHO) detected a large coronal mass ejection (CME) associated with this burst. We find that the $\mathrm{CME}$ onset phase, derived from the limb crossing time after a linear extrapolation back from the height-time coronagraph observations to the solar surface, occurred considerably before the onset time of this large burst. This extrapolation is shown in Figure 1, suggesting that the CME onset phase began at about 19:34 UT, with a \pm 1 min uncertainty (assuming the validity of the linear extrapolation). The figure also shows three data points from the NASA 


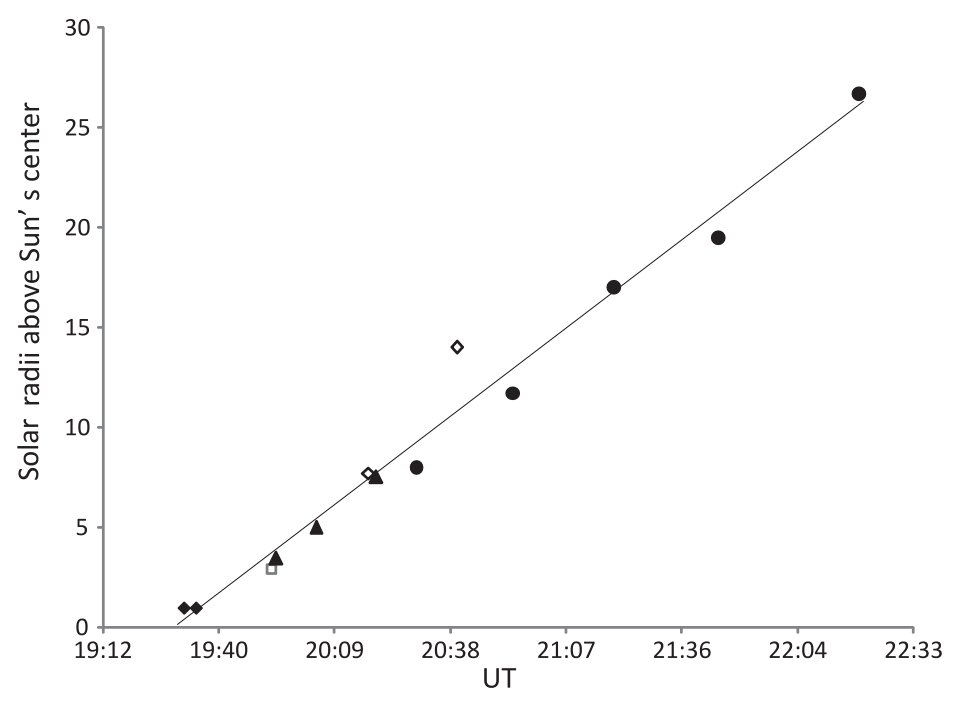

Figure 1 Radial distances of CME features above the solar limb, derived from LASCO C2 (filled triangles) and C3 (filled circles) instruments. The limb crossing time by a linear extrapolation suggests an onset time at about 19:34 UT on 4 November 2003, close to the first $\mathrm{H} \alpha$ brightening observed by BBSO (filled diamonds, see Figure 3). The data from NASA CME catalogue (http://cdaw.gsfc.nasa.gov/CME_list/) have been added, with two positions obtained from C3 (open diamonds) and one from C2 (open square), exhibiting a similar onset time, within the uncertainty of a few minutes, assuming the validity of the linear extrapolation.
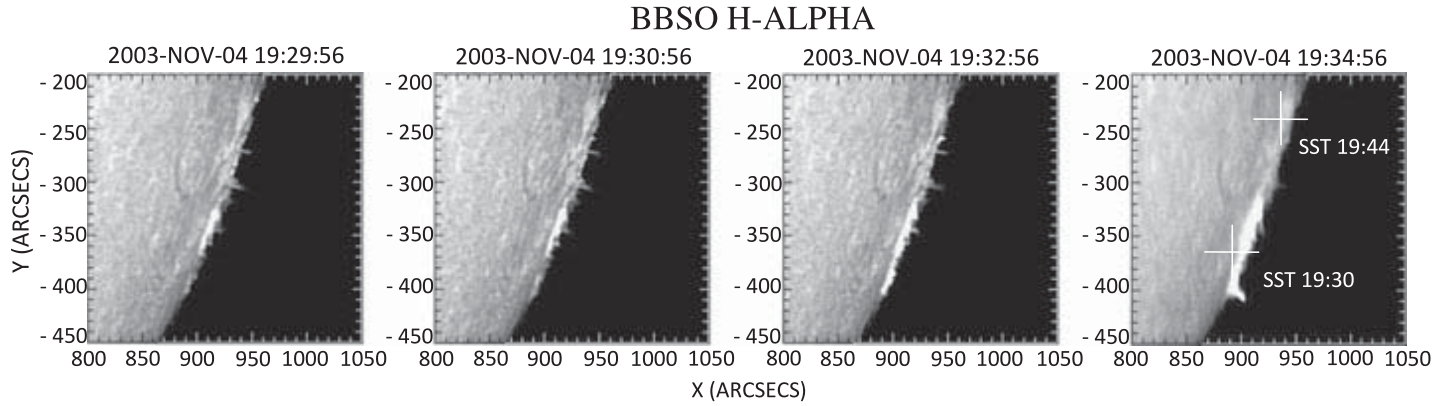

Figure 2 The first H $\alpha$ brightening observed by Big Bear Solar Observatory around 19:32 UT on 4 November 2003. The crosses on the right-most panel indicate the source positions of the sub-THz pulsation at about 19:30 UT and the great flare occurring after 19:40 UT.

CME catalogue (http://cdaw.gsfc.nasa.gov/CME_list/) (which gives a limb crossing time of 19:42 UT or 19:36 UT for rough linear or quadratic extrapolations, respectively). The onset time defined by these extrapolations, however, has an uncertainty due to the limited field of view of the LASCO C2 coronagraph at lower altitudes $\left(\leq 10^{5} \mathrm{~km}\right)$. As noted earlier, it is possible that at altitudes lower than $10^{5} \mathrm{~km}$ an initiation phase, rising with much smaller speed, might have started tens of minutes earlier (Zhang et al., 2001). The presence of the initiation phase could not be directly observed in the LASCO C2 and C3 data analyzed here. On the other hand, the $\mathrm{H} \alpha$ movie obtained by Big Bear Solar Observatory (BBSO, see http:// www.bbso.njit.edu/) shows the very first brightening at about 19:33 - 19:35 UT, close to the suggested CME onset time (see the four successive H $\alpha$ images in Figure 2).

The Reuven Ramaty High Energy Solar Spectroscopic Imager (RHESSI) space experiment (Lin et al., 2002) obtained high quality X-ray observations just before the large burst of 4 November 2003. These data were investigated together with observations obtained at sub-THz frequencies by the Solar Submillimeter Telescope (SST; Kaufmann et 
al., 2008), in microwaves obtained by the solar array at Owens Valley Radio Observatory (OVRO; Gary and Hurford, 1999), and the Itapetinga $7 \mathrm{GHz}$ polarimeter (Kaufmann, 1971; Correia, Kaufmann, and Melnikov, 1999). Our investigation reveals an unusual pulsating and bursting activity, together with a slow, varying enhancement in X-rays, preceding the CME onset. We discuss a possible association between the early CME initiation phase and the major burst.

\section{Multi-wavelength Observations}

In Figure 3 we show the time profiles describing the solar activity 20 min prior to the large burst. The dashed vertical line indicates the approximate CME onset time. GOES $1-8 \AA$ and RHESSI X-ray time profiles are displayed at the top. The GOES soft X-ray flux appeared to rise after about 19:29 UT, nearly the same time as the rise in RHESSI 6-10 keV Xrays. The RHESSI $25-50 \mathrm{keV}$ X-ray flux began to rapidly increase after 19:32 UT. This rapid increase is preceded by two slow oscillations at about 19:27:30 UT and 19:30 UT. The sudden intensity drop just before 19:34 UT (19:33:48 UT) in the 6-10 and 25-50 keV bands is an artifact caused by the automatic insertion of X-ray attenuators. The enhanced count rate in these bands from 19:38:00 to 19:38:28 UT was caused by a brief removal of the attenuators. The RHESSI $100-300 \mathrm{keV}$ flux exhibits one impulsive burst peaking at about 19:34 UT, which is nearly coincident with a microwave impulsive burst (7 GHz flux shown in the bottom of the figure). Both are nearly coincident with the derived CME onset time. These events were followed by another small impulsive burst, detected in the two higher energy RHESSI X-ray bands only, at about 19:36.3 UT. RHESSI was in spacecraft nighttime after 19:39 UT.

The bottom panel of Figure 3 presents the SST data of beams $2(0.2 \mathrm{THz})$ and $5(0.4 \mathrm{THz})$ in units of scintillation index (SI). This index is used to detect the occurrence of pulsations, to facilitate their identification and extraction from the data. It is a normalized, dimensionless magnitude defined as the standard deviation (sigma) of fluctuating data sampled every $40 \mathrm{~ms}$ relative to the signal average taken every $3 \mathrm{~s}$, divided by the difference of the signals obtained by the antennas pointing to the Sun and pointing to the sky. The SI data displayed in Figure 3 have been smoothed by a running mean over $10 \mathrm{~s}$. The pulsations were enhanced around 19:25 UT, with SI exhibiting slow modulations of the order of $1.8 \mathrm{~min}$ at both 0.2 and $0.4 \mathrm{THz}$. The pulsations clearly faded away after the occurrence of the hard X-ray and microwave bursts at about the time of the CME onset or at the beginning of an acceleration phase around 19:34 UT.

The time profile of $7 \mathrm{GHz}$ total flux $(\mathrm{R}+\mathrm{L})$ is also shown in the bottom panel of Figure 3. A small precursor started at about 19:28 UT, together with an increase in the RHESSI Xrays. Two 25-50 keV X-ray enhancements at 19:27:30 and 19:30 UT were observed as the sub-THz pulsations became more pronounced. These were followed by the microwave impulsive burst, and almost simultaneously, by the high-energy RHESSI impulsive event. Both events occurred close to the derived CME onset time (19:35 UT). The microwave spectrum of the impulsive burst had a maximum at about $8 \mathrm{GHz}$ (Figure 4). No clear sub$\mathrm{THz}$ counterpart was observed by SST.

Figure 5 shows the sub- $\mathrm{THz}$ pulsations during a portion of the period of enhanced scintillation index (Figure 3), in the $30 \mathrm{~s}$ interval 19:30:30-19:31:00 UT. The two plots represent SST data of $40 \mathrm{~ms}$ sampling from beams $2(0.2 \mathrm{THz})$ and $5(0.4 \mathrm{THz})$, without any running mean smoothing. Despite the much noisier $0.4 \mathrm{THz}$ data, there is a nearly one-to-one correspondence of quasi-periodic time structures, displaying about 14 time structures in $30 \mathrm{~s}$. 
Figure 3 Multi-wavelength time profiles observed on 4 November 2003 before the onset of the large burst at 19:40 UT. As the GOES and RHESSI soft X-rays increased (upper two plots in the top panel), there was an enhancement of the scintillation indices at two sub-THz

frequencies (bottom panel). Impulsive bursts were observed in hard X-rays and microwaves at about the CME onset time. The sudden drops in RHESSI 6-10 and $25-50 \mathrm{keV}$ signals at about 19:34 UT are instrumental effects.
Figure 4 Spectrum of the microwave impulsive burst that occurred at about 19:34 UT, obtained by the Owens Valley Solar Array.
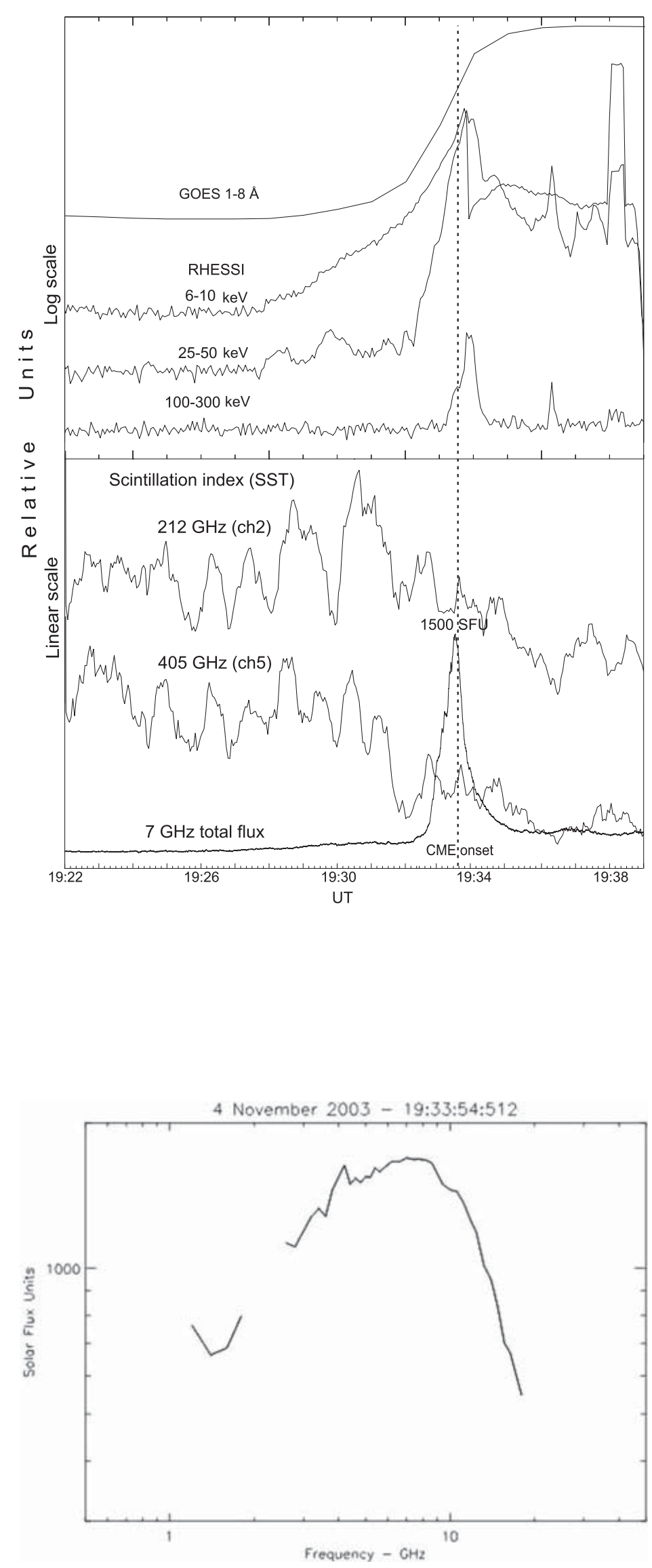


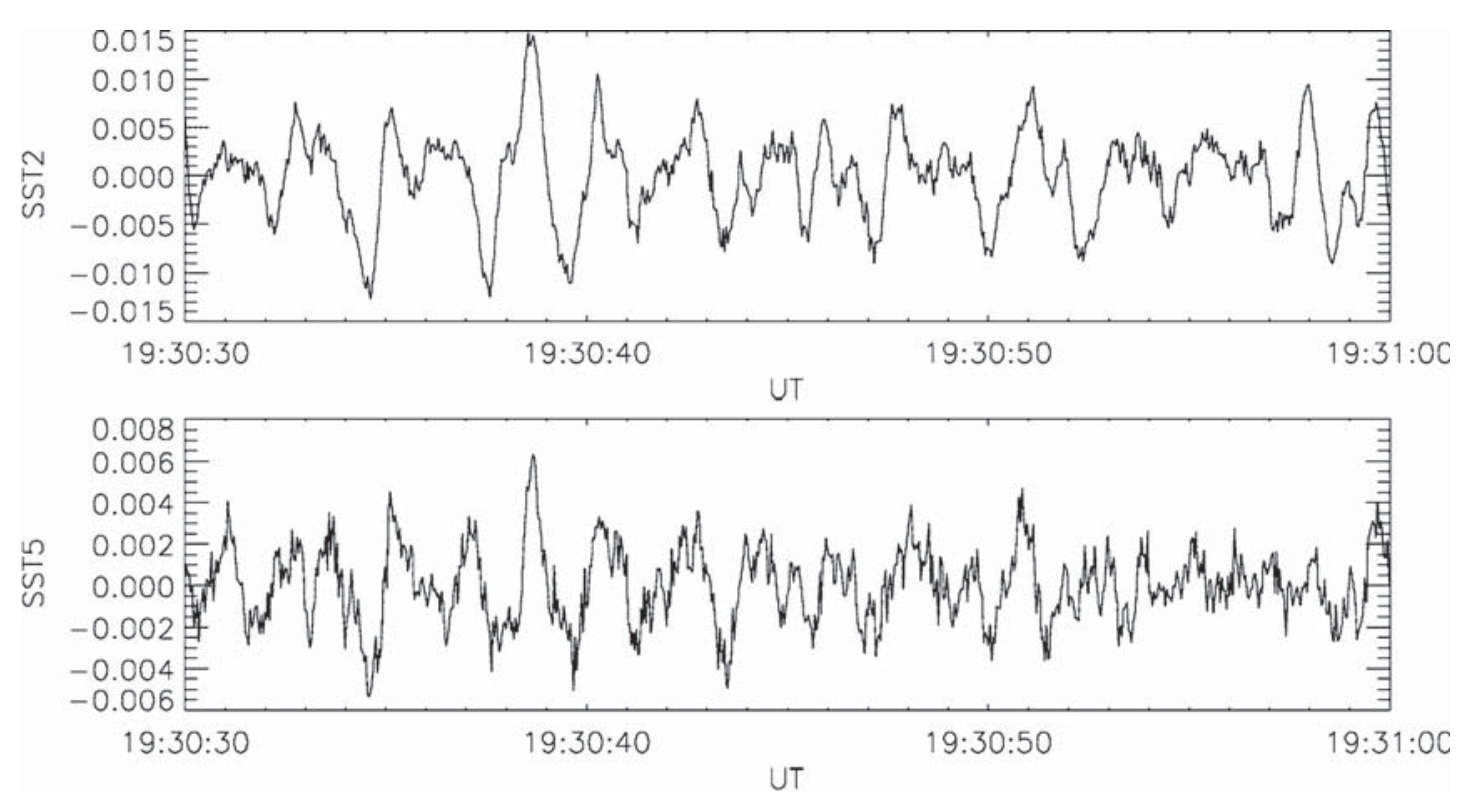

Figure 5 Enlarged SST flux variations showing pulsations observed at 19:30:30 - 19:31:00 UT. There is a nearly one-to-one correspondence between the pulses at 0.2 (top panel) and 0.4 (bottom panel) THz, for about 14 time structures.

\section{Spatial Positions}

The SST has an arrangement of four $0.2 \mathrm{THz}$ and two $0.4 \mathrm{THz}$ feed horns at the focal plane of a $1.5 \mathrm{~m}$ diameter antenna (Kaufmann et al., 2008). The corresponding half-power beamwidths are 4 arcmin and 2 arcmin, at 0.2 and $0.4 \mathrm{THz}$, respectively. Three of the $0.2 \mathrm{THz}$ beams $(2,3$, and 4$)$ are arranged in a triangular configuration and partially overlap with each other with their axes separated by the half-power beamwidth. One of the $0.4 \mathrm{THz}$ beams (beam 5) points at the center of the triangle. This cluster of four beams tracks the solar active center. The other two beams $1(0.2 \mathrm{THz})$ and $6(0.4 \mathrm{THz})$ are co-aligned and displaced by 8 arcmin from the cluster of the four beams (i.e., beams 2,3 , and 4 at $0.2 \mathrm{THz}$ and beam 5 at $0.4 \mathrm{THz}$ ). A comparison of partially overlapping beams at $0.2 \mathrm{THz}$ allows the determination of the burst position in space (Giménez de Castro et al., 1999).

In Figure 6 we show the SST source positions with the crosses on a $195 \AA$ EUV image from SOHO EIT. The size of the crosses indicates the approximate uncertainty of the position determinations which is about \pm 25 arcsec. The source position of the pulsations at 19:30 UT is about 2 arcmin south of the position of the major burst detected nearly $10 \mathrm{~min}$ later (19:40 - 19:50 UT) and very close to the intensity peaks in RHESSI images at 12 $20 \mathrm{keV}$ and $25-50 \mathrm{keV}$, as shown in Figure 6. At an earlier time (19:29:12 UT) the RHESSI source was further north and between the two SST sources indicated by crosses in Figure 6 . The RHESSI source location matched closely the source position of the SST pulsations as they became more pronounced, at about the time of the impulsive bursts in microwaves and $\mathrm{X}$-rays, and the CME onset time (i.e., at 19:33:50 UT). These positions are also close to the first chromospheric $\mathrm{H} \alpha$ brightening shown in Figure 2.

\section{Discussion}

The following remarkable features were found for this event, in the pre-flare phase of the large X28 flare: 


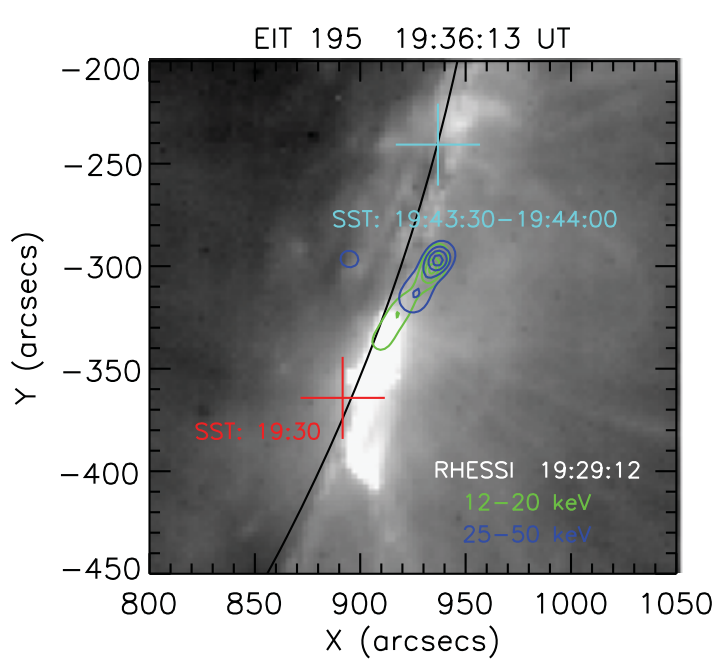

(a)

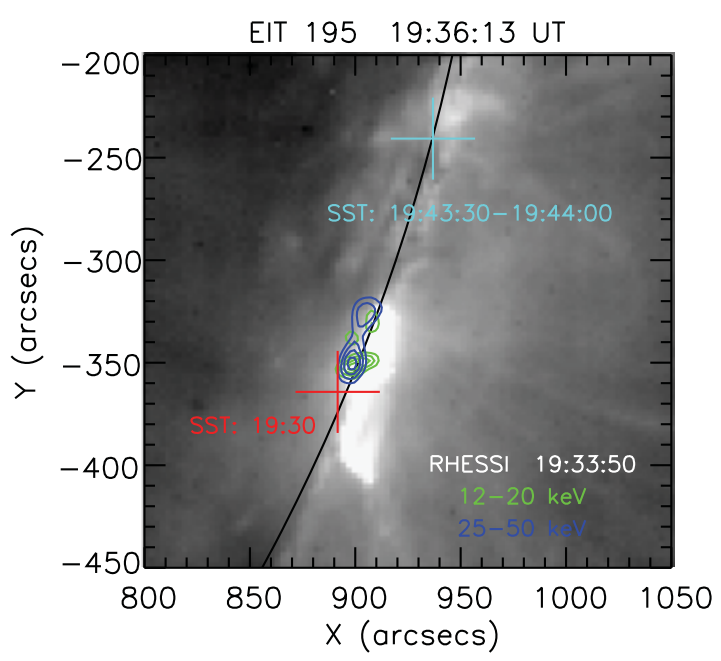

(b)

Figure 6 The RHESSI $12-20$ and 25-50 keV X-ray images and SST source positions overlaid on an EIT $195 \AA$ solar disk image. Crosses show the SST source positions of the pulsations at 19:30 UT (red), and the large burst that occurred later at 19:44 UT (cyan), with uncertainties of \pm 25 arcsec. The RHESSI contours are shown in green $(12-20 \mathrm{keV})$ and blue $(25-50 \mathrm{keV})$. Panel (a), obtained earlier at 19:29:12 UT, shows the X-ray sources located between the two SST crosses. Panel (b), obtained at 19:33:50 UT during the initial impulsive burst and at the derived CME onset time, indicates that the X-ray sources coincide in position with the SST pulsating source, nearly 2 arcmin south of the position of the large burst that began around 19:40 UT.

i) The pulsations of emission at sub-THz frequencies started at least $10 \mathrm{~min}$ before the deduced CME onset time. The pulsations exhibited short periods of $3-5 \mathrm{~s}$, and were modulated by a slower component with periods of about $1-2$ min.

ii) The sub-THz pulsations became more pronounced and the RHESSI $25-50 \mathrm{keV} \mathrm{X-rays}$ showed superimposed time structures as the intensity rose in RHESSI 6-10 keV, GOES soft X-rays, and in microwaves.

iii) As the sub-THz pulsations faded out an impulsive microwave burst occurred together with a RHESSI hard X-ray impulsive burst, at about the CME onset time and the time of the first $\mathrm{H} \alpha$ brightening.

iv) The sub-THz, RHESSI X-ray, and BBSO H $\alpha$ activities occurred before and at about the CME onset time at a different location from that at the large burst which occurred nearly six minutes after the CME onset time.

This detailed description reveals complex energy build up activity in X-rays, H $\alpha$, sub$\mathrm{THz}$ wavelengths, and microwaves. The sub-THz pulsations became more pronounced after about 19:24 UT, together with the first soft X-ray rise observed by GOES and RHESSI. They probably represent physical processes corresponding to the CME initiation phase (Zhang et $a l ., 2001)$ and are related to the subsequent CME onset phenomena, but apparently without a clear connection to the large flare that occurred afterwards. The whole picture fits well the evidence that the CME onset most commonly precedes the flare (Harrison, 1986; Lin, 2005).

The emission mechanism responsible for the sub-THz radiation has not been established. Two possibilities are free-free radiation from hot, thermal plasma and gyrosynchrotron from high-energy, non-thermal electrons in a strong magnetic field (Zirin, 1988; Zirin, MacKinnon, and Mackenna-Lawlor, 1991; and references therein). Either mechanism, however, must be optically thick, since the intensity at $0.4 \mathrm{THz}$ is greater than at $0.2 \mathrm{THz}$. Below we consider implications of each mechanism. 
The X-ray events prior to the CME onset time seem to be due to thermal emission, favoring the suggested mechanism of reconnection with plasma heating and a possible plasmoid ejection leading to the main CME acceleration phase (Ohyama and Shibata 1997, 1998). The sub-THz pulsations, with time scales of a few seconds, might be a response of the magnetically confined and heated thermal plasma to magnetohydrodynamic oscillations associated with resonant Alfvén waves (for example Nitta et al., 1991; Fleishman, Bastian, and Gary, 2008; Kislyakova et al., 2011). These modulations have an important role in producing the RHESSI $25-50 \mathrm{keV}$ time structures, in the time interval of the first soft X-ray enhancement, which represent a response to reconnection episodes at different heights, with possible plasmoid ejections adding to the overall CME evolution in space (Ohyama and Shibata 1998, 1998; Nishizuka et al., 2010). The fading out of the pulsations at the CME onset time might be related to the ultimate release of heated plasma, leaving only a relatively small amount of material, not sufficient to respond to the magnetic field modulations.

The processes occurring during the CME initiation phase and prior to the onset time seem to include the clustering of rapid and successive pulses at sub-THz frequencies. This can be reconciled with other non-thermal mechanisms closer to or at the solar surface, such as the superposition of multiple or fragmented energy releases (Biesecker and Thompson, 2000). This process produces gyrosynchrotron emissions, as indicated by a number of flare diagnostics and model descriptions (Frost, 1969; van Beek, de Feiter, and de Jager, 1974; Kaufmann et al., 1980; Kaufmann, 1985; Lu and Hamilton, 1991; Zirker and Cleveland, 1993; MacKinnon, MacPherson, and Vlahos, 1996). As the magnetic complexity grows in an active region, conditions are reached before a major large-scale instability sets in, inducing multiple and faster instabilities on smaller scale magnetic arcades or fluxules (Sturrock and Uchida, 1981; Sturrock et al., 1984; Sturrock, 1986; and references therein). The energy released by such elementary instabilities produces nanoflares or, when clustered in a larger number in a short time, brighter flares. Models based on larger spatial scale structures describe CMEs arising from the disruption of certain coronal streamer configurations by flux-rope instabilities at the photospheric level (Wu et al., 2000), which can be reconciled with similar scenarios with multiple and complex magnetic structures.

Quasi-periodic pulsations (QPP) with time scales of seconds to tens of seconds obtained at short microwave wavelengths (17 and $34 \mathrm{GHz}$ ) with the Nobeyama Radioheliograph have been studied by Inglis, Nakariakov, and Melnikov (2008). Two groups of generating mechanisms have been proposed (Melnikov et al., 2005; Nakariakov and Melnikov, 2009). The former assumes repetitive regions of flaring energy releases by magnetic reconnection or other means, and the latter assumes coherent MHD oscillations of all regions contributing to the flare emission. We may extend this interpretation to the sub-THz pulsations. The sample shown in Figure 5 indicates that the pulses are in phase at the two frequencies with a reduction in amplitude for the higher $0.4 \mathrm{THz}$ frequency. This may suggest optically thick emission from very dense plasma $\left(\geq 10^{12} \mathrm{~cm}^{-3}\right)$. Adopting the global sausage mode of oscillations in a magnetic tube (Melnikov et al., 2005), the sub-THz radiation possibly originates from the inner, highly dense sections of the tube.

Finally, the assumption of multiple energy releases as the origin of the pulsations can also be reconciled with recent results obtained from simulations of far infrared and sub- $\mathrm{THz}$ thermal continuum emission as a response of denser plasma in the solar atmosphere to timevariable heating by electron beams (Kasparova et al., 2009). They show that the continuum intensities can vary on the same time scale as short-duration variations in the electron beam flux, from sub-second to longer durations. 


\section{Conclusions}

We analyzed in detail the activities 20 min before the large burst of the 4 November 2003 flare with instruments at multiple wavelengths: X-rays by RHESSI, LASCO coronagraphs on the SOHO spacecraft, $\mathrm{H} \alpha$ by BBSO, two sub-THz frequencies by SST, and high sensitivity Itapetinga polarimeter at $7 \mathrm{GHz}$, and at multiple microwave frequencies by OVRO. The first enhancements at soft X-rays and microwaves were observed nearly 10 min before the CME onset. We also found the sub-THz pulsations nearly 6 min before the onset of the largest X-class flare.

The CME onset time coincided with an impulsive burst in hard X-rays and microwaves with moderate intensity, nearly $6 \mathrm{~min}$ before the large burst onset. The CME onset time was preceded by pulsations of 3-5 s periods at sub-THz frequencies that began nearly 10 min earlier, together with soft X-ray and $7 \mathrm{GHz}$ enhancements and superimposed 25 $50 \mathrm{keV} \mathrm{X}$-ray time structures. These unusual phenomena preceding the CME onset strongly indicate that the CME material started to be lifted up at least 10 min before a sudden energy release occurs and the CME is accelerated strongly upward (Ohyama and Shibata 1998, 1998; Nishizuka et al., 2010).

The position of the X-ray source corresponded to the approximate position of the sub$\mathrm{THz}$ pulsations before and at the CME onset time, when the first $\mathrm{H} \alpha$ brightening appeared, all displaced by about 2 arcmin south from the position of the large flare that occurred later, suggesting they were distinct events. The sub-THz pulsations faded out as impulsive bursts were observed in X-rays and microwaves at about the same time as the CME onset. The complex, unusual activity before and at the CME onset time might be representative of the initiation phase preceding the sudden acceleration of the ejected material (Zhang et al., 2001).

The physical mechanism producing the sub-THz pulsations is still unclear. To improve our understanding, more observational and theoretical investigations are required. Existing developments are potentially helpful in the search for explanations. For example, we may extend to sub-THz frequencies the adoption of two groups of mechanisms proposed to explain short microwave (i.e., 17 and $34 \mathrm{GHz}$ ) QPP pulsations (Melnikov et al., 2005; Nakariakov and Melnikov, 2009). One assumes multiple flaring energy releases, and another assumes MHD-modulated plasma emission. The response to MHD sausage-mode modulation of a confined plasma also has an important role in producing the RHESSI $25-50 \mathrm{keV}$ time structures. This in turn represents a response to reconnection episodes at different heights, with plasmoid ejections adding to the overall CME evolution in space (Ohyama and Shibata 1998, 1998; Nishizuka et al., 2010). The sub-THz pulsations during the CME initiation phase can also be reconciled with suggested mechanisms closer to or at the solar surface, such as the superposition of multiple or fragmented energy release events. As the magnetic complexity grows in an active region, even before a major largescale instability sets in, multiple and faster instabilities may appear in smaller scale magnetic arcades or fluxules (for example, Sturrock and Uchida, 1981; Sturrock et al., 1984; Sturrock, 1986). Recent results from simulations show rapid pulsations in far infrared and sub-THz thermal continuum emission as the response of denser plasma in the solar atmosphere to time-variable heating by electron beams with sub-second or longer durations (Kasparova et al., 2009).

A number of known CME-related solar activities were confirmed in this event, namely the onset of soft X-rays, the pronounced sub-THz pulsations before the CME onset time, and the close time coincidence between the CME onset and the earlier, smaller impulsive burst. New features were found here, such as the slow modulation (tens of seconds) of sub- $\mathrm{THz}$ 
pulsations (seconds), which are somewhat related to enhancements in soft X-rays and time structures in harder RHESSI X-rays. The pulsations seemed to fade away once the CME was launched. The relative positions in space of the X-ray source, the sub-THz pulsations, and first $\mathrm{H} \alpha$ brightening before and at the CME onset, displaced by about 2 arcmin south from the position of the large flare that occurred later, suggests that they were distinct events.

The overall scenario with respect to the large burst that occurred later can be well described by the qualitative picture (Harrison, 1986) of a CME loop expansion triggering a major flare away from the centerline of material ejection, tens of minutes after its launch time.

New patterns associated with the CME occurrence were obtained from the combined analysis of the low-level X-ray activity observed by RHESSI together with sub-THz and microwave pulsations. Analysis of a larger number of events, for which there is a similar combination of data available, should reveal new clues about their physical nature and the links between them, which are not currently well understood.

Acknowledgements We wish to thank an anonymous referee for very helpful comments. We acknowledge Dr. Dale Gary for providing the OVSA microwave burst spectrum. Gordon Holman and Yang Su acknowledge support from the RHESSI project and a NASA Heliophysics Guest Investigator Grant. This research was partially supported by the Brazilian agencies FAPESP, CNPq, Mackpesquisa, Argentina agency CONICET, and US agency AFOSR.

\section{References}

Brodrick, D., Tingay, S., Wieringa, M.: 2005, J. Geophys. Res. 110, A09S36.

Brueckner, G.E., Howard, R.A., Koomen, M.J., Korendyke, C.M., Michels, D.J., Moses, J.D., et al.: 1995, Solar Phys. 162, 357.

Correia, E., Kaufmann, P., Melnikov, V.: 1999, In: Bastian, T.S., Gopalswamy, N., Shibasaki, K. (eds.) Solar Physics with Radio Observations, Proceedings of the Nobeyama Symposium, Nobeyama Radio Observatory Report 479, 263.

Datlowe, D.W., Elcan, M.J., Hudson, H.S.: 1974, Solar Phys. 39, 155.

Fleishman, G.D., Bastian, T.S., Gary, D.E.: 2008, Astrophys. J. 864, 1433.

Frost, K.J.: 1969, Astrophys. J. Lett. 158, L159.

Gary, D.E., Hurford, G.J.: 1999, In: Bastian, T.S., Gopalswamy, N., Shibasaki, K. (eds.) Solar Physics with Radio Observations, Proceedings of the Nobeyama Symposium, Nobeyama Radio Observatory Report 479, 429.

Giménez de Castro, C.G., Raulin, J.-P., Makhmutov, V.S., Kaufmann, P., Costa, J.E.R.: 1999, Astron. Astrophys. Suppl. Ser. 140, 373.

Gopalswamy, N., Mikić, Z., Maia, D., Alexander, D., Cremades, H., Kaufmann, P., Tripathi, D., Wang, Y.-M.: 2006, Space Sci. Rev. 123, 303.

Harrison, R.A., Waggett, P.W., Bentley, R.D., Phillips, K.J.H., Bruner, M., Dryer, M., Simnett, G.M.: 1985, Solar Phys. 97, 387.

Harrison, R.A.: 1986, Astron. Astrophys. 162, 283.

Inglis, A.R., Nakariakov, V.M., Melnikov, V.F.: 2008, Astron. Astrophys. 487, 1147.

Kahler, S.W.: 1992, Astron. Astrophys. 30, 113.

Kaufmann, P.: 1971, Solar Phys. 18, 336.

Kaufmann, P., Strauss, F.M., Opher, R., Laporte, C.: 1980, Astron. Astrophys. 87, 58.

Kaufmann, P.: 1985, Solar Phys. 102, 97.

Kaufmann, P., Giménez de Castro, C.G., Makhmutov, V.S., Raulin, J.-P., Schwenn, R., Levato, H., Rovira, M.: 2003, J. Geophys. Res. 108(A8), SSH-5-1.

Kaufmann, P., Raulin, J.P., Giménez de Castro, C.G., Levato, H., Gary, D.E., et al.: 2004, Astrophys. J. Lett. 603, L12.

Kasparova, J., Heinzel, P., Karlicky, M., Moravec, Z., Varady, M.: 2009, Cent. Eur. Astrophys. Bull. 33, 309.

Kaufmann, P., Levato, H., Cassiano, M.M., Correia, E., Costa, J.E.R., Giménez de Castro, C.G., et al.: 2008, In: Stepp, L.M., Gilmozzi, R. (eds.) Ground-based and Airborne Telescopes II, Proc. SPIE 7012, 70120L. 
Kislyakova, K.G., Zaitzev, V.V., Altyntsev, A.T., Meshalkina, N.S.: 2011, In: European Week of Astronomy and Space Science, JENAM-2011, Book of Abstracts, 41.

Lin, R.P.: 2005, Adv. Space Res. 35, 1857.

Lin, R.P., Dennis, B.R., Hurford, G.J., Smith, D.M., Zehnder, A., Harvey, P.R., et al.: 2002, Solar Phys. 210 , 3.

Lu, E.T., Hamilton, R.J.: 1991, Astrophys. J. Lett. 380, L89.

MacKinnon, A.L., MacPherson, K.P., Vlahos, L.: 1996, Astrophys. J. Lett. 310, L9.

Melnikov, V.F., Reznivova, V.E., Shibasaki, K., Nakariakov, V.M.: 2005, Astron. Astrophys. 439, 727.

Mittal, N., Narain, U.: 2010, J. Atmos. Solar-Terr. Phys. 72, 643.

Nakariakov, V.M., Melnikov, V.F.: 2009, Space Sci. Rev. 149, 119.

Nishizuka, N., Takasaki, H., Asai, A., Shibata, K.: 2010, Astrophys. J. 711, 1062.

Nitta, N., White, S.M., Kundu, M.R., Gopalswamy, N., Holman, G.D., Brosius, J.W., Schmelz, J.T., Saba, J.L.R., Strong, K.T.: 1991, Astrophys. J. 374, 374.

Ohyama, M., Shibata, K.: 1997, Publ. Astron. Soc. Japan 49, 5.

Ohyama, M., Shibata, K.: 1998, Astrophys. J. 499, 934.

Schwenn, R., Raymond, J.C., Alexander, D., Ciaravella, A., Gopalswamy, N., Howard, R., et al.: 2006, Space Sci. Rev. 123, 127.

Sturrock, P.A., Uchida, Y.: 1981, Astrophys. J. 246, 331.

Sturrock, P.A., Kaufmann, P., Moore, R.L., Smith, D.F.: 1984, Solar Phys. 94, 341.

Sturrock, P.A.: 1986, In: Dennis, B.R., Orwig, L.E., Kiplinger, A.L. (eds.) Proc. SMM Topical Workshop on Rapid Fluctuations in Solar Flares, NASA CP-2449, 1.

Thomson, N.R., Rodger, C.J., Dowden, R.L.: 2004, Geophys. Res. Lett. 31, L06803.

van Beek, H.F., de Feiter, L.D., de Jager, C.: 1974, Space Res. XIV, 447.

Webb, D.F.: 2000, J. Atmos. Solar-Terr. Phys. 62, 1415.

Woods, T.N., Acton, L.W., Bailey, S., Eparvier, F., Garcia, H., Judge, D., et al.: 2004, Geophys. Res. Lett. 31, L10802.

Wu, S.T., Guo, W.P., Plunkett, S.P., Schmieder, B., Simnett, G.M.: 2000, J. Atmos. Solar-Terr. Phys. 62 , 1489.

Zhang, J., Dere, K.P., Howard, R.A., Kundu, M.R., White, S.M.: 2001, Astrophys. J. 559, 452.

Zirin, H.: 1988, In: Astrophysics of the Sun, Cambridge University Press, New York, 343 - 404.

Zirin, H., MacKinnon, A., Mackenna-Lawlor, S.M.: 1991, In: Cox, A.N., Livingston, W.C., Mathews, M.S. (eds.) Solar Interior and Atmosphere, The University of Arizona Press, Tucson, 964-995.

Zirker, J.B., Cleveland, F.M.: 1993, Solar Phys. 144, 341. 